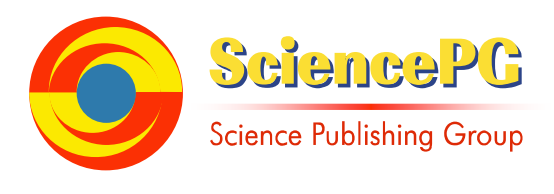

\title{
Improving Cognitive Impairment of Elderly by an Educational Program for Their Caregivers
}

\author{
Omneya Mohamed ${ }^{1}$, Amany Sorour ${ }^{2}$, Amany Aboelseoud ${ }^{3}$, Rasha Fahmy ${ }^{4}$ \\ ${ }^{1}$ Community Health Nursing Department, Faculty of Nursing, Zagazig University, Zagazig, Egypt \\ ${ }^{2}$ Community Health Nursing Department, Faculty of Nursing, Zagazig University, Cairo, Egypt \\ ${ }^{3}$ Community Health Medicine Department, Faculty of Medicine, Zagazig University, Cairo, Egypt \\ ${ }^{4}$ Neurology Department, Faculty of Medicine, Zagazig University, Zagazig, Egypt

\section{Email address:} \\ dr.amany.sorour@gmail.com (A. Sorour)
}

\section{To cite this article:}

Omneya Mohamed, Amany Sorour, Amany Aboelseoud, Rasha Fahmy. Improving Cognitive Impairment of Elderly by an Educational Program for their Caregivers. American Journal of Nursing Science. Vol. 4, No. 6, 2015, pp. 317-323. doi: 10.11648/j.ajns.20150406.14

\begin{abstract}
Cognitive impairment is associated with increased risk for progression to dementia and functional disability. The aim of this study was to examine the effectiveness of an educational program for the caregivers of elderly persons in improving their cognitive impairment. This quasi-experimental design with pre-post assessment was conducted at four geriatric care homes in Cairo governorate on 34 elderly persons having cognitive impairment (Mini-Mental State Examination [MMSE] score 19 to 24). The researchers prepared the training program for the caregivers. It included knowledge about dementia, instructions to deal with the elderly persons, and a practical part focused on memory aids. The effectiveness of the program was measured by comparing the weekly morning and evening MMSE scores, and comparing the baseline (pre-test) and 8-week (post-test) MMSE scores. The data was from August 2012 to April 2013. The results showed statistically significant improvements in elderly's MMSE scores $(p<0.001)$. The total MMSE score improved by 3.50 points. All elderly had cognitive impairment before the intervention; this decreased to $29.4 \%$ after the intervention $(\mathrm{p}<0.001)$. The improvement was markedly higher in the morning measurements compared with the evening ones. In multivariate analysis, the intervention was a statistically significant independent predictor of the improvement in the total MMSE score, in addition to the educational level. In conclusion, training the caregivers of elderly people in mental stimulation and memory aids can improve the cognitive impairment among these persons. The findings corroborate the need for developing intervention programs that equip the caregivers of elderly people with non-pharmacological management strategies that enable them to improve their cognitive impairment. Further research is needed to investigate the effectiveness of memory aids in improving the memory of elderly at different stages of dementia.
\end{abstract}

Keywords: Cognitive Impairment, Mini-Mental State Examination, Elderly, Caregiver

\section{Introduction}

Age-related decline in episodic memory, attention, and executive function is reported in both longitudinal [1] and cross-sectional [2] studies. Cognitive impairment that does not reach the threshold for dementia diagnosis is not only associated with increased risk for progression to dementia [3], but also increased functional disability [4]. The high prevalence of cognitive impairment with advancing age, together with rapid demographic ageing, underlines the importance of developing interventions to improve or maintain cognitive function in later life [5].

Mild cognitive impairment (MCI) is sometimes considered a precursor of dementia or as the boundary between normal aging and dementia [6]. The prevalence of $\mathrm{MCI}$ is between $10 \%$ and $20 \%$ among people over 65 years of age $[7,8]$. The criteria set for MCI diagnosis are i) the person is neither normal nor demented; ii) there is evidence of cognitive deterioration shown by either objectively measured decline over time and/or a subjective report of decline by self and/or informant in conjunction with objective cognitive deficits; and iii) activities of daily living are preserved and complex instrumental functions are either intact or minimally impaired [9].

Interventions comprising modifiable lifestyle factors, such as cognitive, social, and physical activity, that may reduce the risk of cognitive decline have been gaining increasing interest 
$[10,11]$. Of these strategies, cognitive interventions are specifically targeted at improving cognitive performance. Cognitive training comprises specifically designed training programs that provide guided practice on a standard set of cognitive tasks, aimed at improving performance in one or more cognitive domains [12]. While a number of randomized controlled trials have shown that cognitive training can improve cognitive performance in healthy older adults [13], improvements often do not exceed those seen in active control conditions [12].

General mental stimulation refers to interventions that promote increased engagement in mentally stimulating activities. Examples include activities that might be undertaken by individuals as part of daily living such as reading, playing music or playing chess. Epidemiological evidence suggests that higher levels of engagement in mental stimulation are associated with lower rates of cognitive decline particularly in working memory and processing speed [14]. However, most of the evidence to date is correlational and only a limited number of true experimental intervention studies have examined the efficacy of mental stimulation on cognition. Therefore, the aim of this study was to examine the effectiveness of an educational program for the caregivers of elderly persons in improving their cognitive impairment.

\section{Subjects and Methods}

Research design and setting: A quasi-experimental design with pre-post assessment was used to achieve the aim of the study, which was conducted at four geriatric care homes in Cairo governorate (El-maha, Koloub el-kheir, El-kamilia, and El-habaieb).

Subjects: A convenience sample of 34 elderly persons residing in the aforementioned settings was recruited. The inclusion criteria were being 60 -year age or older, having cognitive impairment diagnosed by a Mini-Mental State Examination (MMSE) test score 19 to 24, and having a caregiver willing to participate in the study. Those with debilitating conditions making them not able to participate in the program or to respond were excluded. The sample size was calculated to estimate an improvement of at least 0.5 point in the Mini-Mental State Examination (MMSE) test score, at $95 \%$ level of confidence and $80 \%$ power, using the sample size equation for paired t-test in Epi-Info program.

Data collection tool: The researcher used an interview questionnaire sheet composed of two parts. The first part was concerned with respondent's personal data such as age, gender, educational level, family number, and job status. The second part consisted of the standardized Mini-Mental State Examination (MMSE) scale developed by Folstein et al [15]. It is a valid and reliable instrument used to screen cognitively impaired elderly people. A standardized approach to scoring and interpreting older people's cognitive functions provides a global score of cognitive ability that correlates with daily functions.

The scale consists of 30 statements with true/false responses categorized in six domains. The orientation domain measures person's orientation about time and place. The registration domain examines the ability repeat the names of three objects mentioned by the examiner. In the attention and calculation domain, the person is asked to subtract 7 from 100 then repeat from result, and stop after five subtractions; alternatively, if the person has errors in subtracting, he/she is asked to spell one word forward and then backward. In the recall or recording domain, the person is asked to name the three objects learned earlier. In the language, the person is asked to name two objects such as a pencil and a watch. The repeat domain was excluded since many of the elders could not read or write. In the last domain of copying, the person is asked to copy intersecting pentagons. The responses are scored one for correct answer and zero for incorrect answer. The score are summed-up for each domain and for the total scale. A total score of less than 25 indicates cognitive impairment.

Pilot study: A pilot study was carried out on 5 patients representing about $10 \%$ of the total study sample over a period of two months. The aim was to test feasibility of the study, and the exact time required for filling the questionnaire sheet. Since no changes were done in the tools, the participants involved in the pilot were included in the main study sample.

Fieldwork: After obtaining the official permission to conduct the study and after finalization of tools, the study was carried out through assessment, planning, implementation, and evaluation phases.

Assessment phase: The researcher met with the caregivers of the elderly persons to explain the purpose and the procedure of the study, and invited them to participate. Those who gave their consent to participate were interviewed individually. Personal and baseline data about the level of cognitive impairment were obtained about the elderly persons using the interview form with the MMSE. Out of 60 elderly assessed, 34 fulfilled the inclusion criterion of a MMSE score between 19 and 24.

Planning phase: During this phase, the researcher prepared the training program for the caregivers. It included a section for basic knowledge about dementia such as definition, risk factors, clinical presentation, and management. It also addressed the instructions to deal with the elderly persons concerning nutrition, depression, and follow-up and its importance. The second section of the program was for practical memory aids. This included simple games to promote memory such as simple mathematics (ascending/descending counting, counting in pairs, descending counting by decrease of five each time, etc.). It also involved identifying simple pictures (phone, car, etc.), naming the seasons of the year, days of the week, months of the year (Gregorian and Higri), the names children and young children, and identifying direct colors. Other exercises included completing songs or familiar proverbs, complete the missing numbers, naming a plant or a country starting with a mentioned letter, etc. A practice exercise consisted of showing the person three unrelated objects, then hiding one and asking him/her to recall it. 
Implementation phase: The researcher implemented the training program to caregivers in small groups. There were short theoretical sessions aimed at giving them basic knowledge about dementia. Then, the practical sessions involved actual training on the various mental exercises included in the program. The attendants rehearsed the exercises on each other and then each on his/her elder demented relative. Then, the caregivers were trained in administering the MMSE test and recording its scoring. The program was provided to caregivers on three sessions, each session lasted from 60 to 90 minutes. They were given a guide booklet prepared by the researcher containing basic knowledge about dementia and related exercises. Each caregiver was provided with a diary to document the frequency and duration of performing the daily exercises. They were asked to document the scores of the MMSE weekly in the morning and evening times. The researcher followed-up the application of the program by each caregiver by phone twice weekly for eight weeks. The data collection took approximately nine months, from August 2012 to April 2013.

Evaluation phase: The researcher collected all the diaries by the end of the 8-week follow-up. The effectiveness of the program was measured by comparing the weekly morning and evening MMSE scores, and comparing the baseline (pretest) and 8-week (post-test) MMSE scores.

Administrative and ethical considerations: The pertinent committees at the Faculty of Nursing, Zagazig University approved the study protocol. Informed consents were obtained from the management of selected geriatric homes and from individual patients and/or caregivers after explaining the aim and objectives of the study as well as the procedures. The researcher clarified the rights to refuse or withdraw at any time without giving reasons, and ensured the confidentiality of any obtained information.

Statistical analysis: Data entry and statistical analysis were done using SPSS 20.0 statistical software package. Quantitative continuous data were compared using paired ttest for repeated measurements. For multiple group comparisons of quantitative data, one-way analysis of variance test (ANOVA) was used. Qualitative categorical variables were compared using chi-square test. In order to identify the independent predictors of MMSE score, multiple linear regression analysis was used after testing for normality, and homoscedasticity and analysis of variance for the full regression models were done. Statistical significance was considered at $\mathrm{p}$-value $<0.05$.

\section{Results}

The study sample included 34 elders whose age ranged between 62 and 72 years, with more males (70.6\%) as shown in Table 1. Approximately one-third had no formal education, while $20.6 \%$ had university education. The majority $(79.4 \%)$ was previously working. The number of other family members ranged between zero and six, with median 4.9.
Table 1. Socio-demographic characteristics of the elderly in the study sample $(n=34)$.

\begin{tabular}{lll}
\hline & Frequency & Percent \\
\hline Age: & & \\
$<70$ & 26 & 76.5 \\
$70+$ & 8 & 23.5 \\
Range & $62-72$ & \\
Mean \pm SD & $67.4 \pm 6.2$ & \\
Median & 67.5 & \\
Gender: & & \\
Male & 24 & 70.6 \\
Female & 10 & 29.4 \\
Educational level: & & \\
None & 10 & 29.4 \\
Basic/intermediate & 17 & 50.0 \\
University & 7 & 20.6 \\
Previous job status: & & \\
Unemployed/housewife & 7 & 20.6 \\
Working & 27 & 79.4 \\
Family size: & & \\
$<5$ & 9 & 26.5 \\
$5+$ & 25 & 73.5 \\
Range & $0-6$ & \\
Mean \pm SD & $5.0 \pm 1.1$ & \\
Median & 4.9 & \\
\hline
\end{tabular}

Table 2 demonstrates statistically significant improvements in participant elderly's Mini-Mental State Examination (MMSE) scores $(\mathrm{p}<0.001)$. The pre-post differences ranged between 0.29 for the domains of registration and copying to 0.88 for the domain of orientation. The total MMSE score improved by 3.50 points.

Table 2. Post-pre-intervention changes in cognitive and MMSE scores of the elderly in the study sample $(n=34)$.

\begin{tabular}{|c|c|c|c|c|c|c|}
\hline & \multicolumn{2}{|c|}{$\begin{array}{l}\text { Post-pre } \\
\text { paired } \\
\text { differences }\end{array}$} & \multicolumn{2}{|c|}{$\begin{array}{l}95 \% \text { Confidence } \\
\text { Interval of the } \\
\text { Difference }\end{array}$} & \multirow[t]{2}{*}{$\begin{array}{l}\text { Paired } \\
\text { t-test }\end{array}$} & \multirow[t]{2}{*}{ p-value } \\
\hline & Mean & SD & Lower & Upper & & \\
\hline \multicolumn{7}{|l|}{$\begin{array}{l}\text { Cognitive } \\
\text { domains }\end{array}$} \\
\hline Orientation & 0.88 & 0.69 & 0.64 & 1.12 & 7.500 & $<0.001^{*}$ \\
\hline Registration & 0.29 & 0.46 & 0.13 & 0.46 & 3.708 & $0.001 *$ \\
\hline Attention & 0.56 & 0.75 & 0.30 & 0.82 & 4.366 & $<0.001 *$ \\
\hline Recording & 0.65 & 0.49 & 0.48 & 0.82 & 7.778 & $<0.001 *$ \\
\hline Language & 0.82 & 0.52 & 0.64 & 1.01 & 9.225 & $<0.001^{*}$ \\
\hline Copying & 0.29 & 0.46 & 0.13 & 0.46 & 3.708 & $0.001 *$ \\
\hline $\begin{array}{l}\text { Total MMSE } \\
\text { score }\end{array}$ & 3.50 & 1.11 & 3.11 & 3.89 & 18.422 & $<0.001 *$ \\
\hline
\end{tabular}

As illustrated in Table 3, all the elderly had cognitive impairment before the intervention. This decreased to $29.4 \%$ after the intervention, and the difference was statistically significant $(\mathrm{p}<0.001)$.

Figure 1 displays a weekly improvement in elderly's MMSE scores throughout the 8-week follow-up $(\mathrm{p}<0.001)$. It is noticed that the improvement was markedly higher in the morning measurements compared with the evening ones.

In multivariate analysis (Table 4), the regression analysis identified that the intervention was a statistically significant 
independent predictor of the improvement in the total MMSE score, in addition to the educational level. However, as the standardized coefficients indicate, the effect of the intervention is higher compared with that of the educational level. Meanwhile, the female gender is a negative predictor of the improvement of the MMSE score. The model explains $80 \%$ of this improvement. Other elderly characteristics as age, job status, and family size have no significant influence on the MMSE score.
Table 3. Post-pre-intervention changes in cognitive impairment (according to MMSE score level) among the elderly in the study sample $(n=34)$.

\begin{tabular}{lclll}
\hline & \multicolumn{2}{c}{ Pre $(\mathbf{n}=34)$} & \multicolumn{2}{l}{ Post $(\mathbf{n}=\mathbf{3 4})$} \\
\cline { 2 - 5 } & No. & \% & No. & \% \\
\hline Cognitive impairment & & & & \\
No & 0 & 0.0 & 24 & 70.6 \\
Yes & 34 & 100.0 & 10 & 29.4 \\
Chi-square test: $37.09, \mathrm{p}<0.001 *$ & & & \\
\hline
\end{tabular}

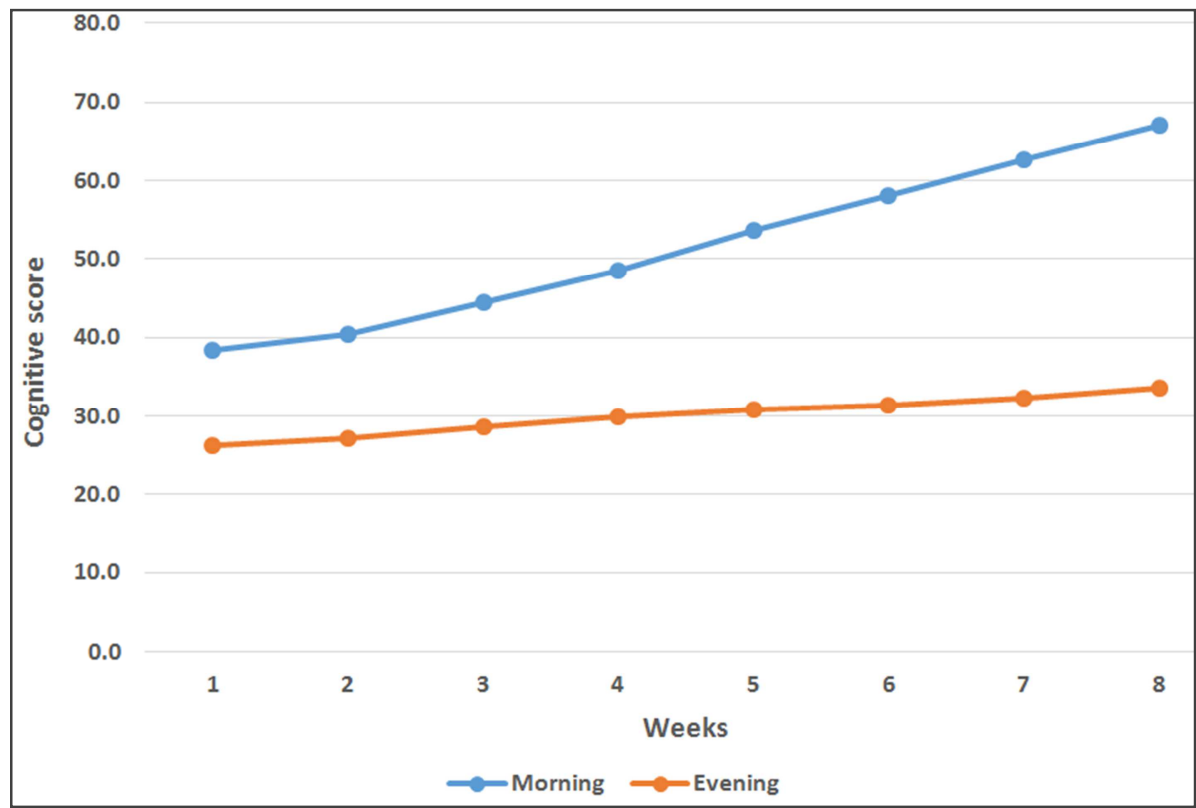

Figure 1. Weekly changes in morning and evening cognitive scores among the elderly in the study sample ( $n=34)$.

Morning scores ANOVA $(\mathrm{F}=59.274, \mathrm{p}<0.001)$

Evening scores ANOVA $(\mathrm{F}=9.410, \mathrm{p}<0.001)$

Table 4. Best fitting multiple linear regression model for the MMS score throughout the intervention.

\begin{tabular}{|c|c|c|c|c|c|c|c|}
\hline & \multicolumn{2}{|c|}{ Unstandardized Coefficients } & \multirow{2}{*}{$\begin{array}{l}\text { Standardized } \\
\text { Coefficients }\end{array}$} & \multirow{2}{*}{ t-test } & \multirow{2}{*}{ p-value } & \multicolumn{2}{|c|}{ 95\% Confidence Interval for B } \\
\hline & B & Std. Error & & & & Lower & Upper \\
\hline Constant & 20.58 & 0.64 & & 32.208 & $<0.001$ & 19.30 & 21.85 \\
\hline Intervention & 3.50 & 0.29 & 0.67 & 12.084 & $<0.001$ & 2.92 & 4.08 \\
\hline Female gender & -3.36 & 0.32 & -0.58 & -10.464 & $<0.001$ & -4.00 & -2.72 \\
\hline Educational level & 1.42 & 0.32 & 0.25 & 4.422 & $<0.001$ & 0.78 & 2.06 \\
\hline
\end{tabular}

r-square $=0.80$

Model ANOVA: $\mathrm{F}=88.28, \mathrm{p}<0.001$

Variables entered and excluded: age, previous job status, family size

\section{Discussion}

Dementia poses major physical and emotional challenges for patients, families, and caregivers, as well as the health care system and the community. Although dementia is more common in advanced age, it is not a normal process of aging [16]. The prevalence of cognitive impairment will double in the next two decades, making it early diagnosis and management a must [17].This study carried out to assess the effect of training the caregivers of elderly persons having cognitive impairment on improving their impairment. The study findings point to a success of this training, with acceptance of the set research hypothesis.

According to the present study findings, there were some variations in the extent of improvement among the different domains of cognitive impairment as assessed by the MMSE. The domains with the highest improvements were those of orientation and language. The improvement in orientation may be attributed to the concomitant improvement in all MMSE memory domains, which increase the orientation of the elderly. In agreement with this, a study in the United Kingdom (UK) revealed that the domain of orientation was 
the most positively influenced by cognitive stimulation interventions in mild dementia [18].On the same line, an intervention study in Australia by Lowenstein et al [19] using cognitive exercises combining specific cognitive techniques as a cognitive rehabilitation treatment for mildly impaired demented persons showed significant improvements in their orientation.

Similarly, the improvement in the language domain was considerable in the current study. This was evident in the abilities of matching, critical judgment and narrative writing. The marked improvement could be explained by the effect of deep learning as practiced in the intervention program. In congruence with this, a randomized clinical trial in Germany demonstrated significant improvements in the language ability of demented elderly patients secondary to an intervention combining physical and mental stimuli [20]. The improvement in language abilities is of great importance since it is one of the best predictors of high cortical functions, and it has a major impact on competency and decisionmaking in dementia [21]. Moreover, Tsantali et al [22], whose findings are similar to ours concerning the improvement in language abilities by cognitive exercises suggested that such interventions could even reverse the progress of dementia.

Nevertheless, the present study revealed less marked improvements in elderly's registration and copying MMSE domains, although they were both statistically significant. This might be due to the high percentage of participants with no formal education, which may have a negative influence on copying abilities. Added to this is the ability of the MMSE to diagnose memory deficits. In this respect, Lacy et al [23] questioned the sensitivity of the MMSE test as a screening tool for memory and recall problems in the elderly. The authors concluded that more in-depth memory testing appears to be a vital component of successful screening and early detection of memory and recall deficits.

Overall, the present study revealed clinically and statistically significant improvements of the total MMSE score among elderly persons with cognitive impairment after the intervention program. The positive and independent impact of the intervention was confirmed through multivariate analysis. This success of the intervention may be attributed the deep learning effects achieved during the encoding and recall procedures as suggested from the literature. Additionally, the training and relearning strengthened consolidation of the benefits gained from the intervention. In line with this, Haslam et al [24] showed that cognitive exercises applying the principles of reality orientation, cognitive stimulation and/or reminiscence therapy are effective in maintaining and improving language, memory and activities of daily living for people with dementia. Even more, the effectiveness of such training was confirmed through showing brain activity changes by MRI imaging $[25,26]$.

The foregoing present study results are in agreement with the study conducted by Cruz et al [27] in Brazil where improved cognition shown by MMSE was demonstrated at a three-month follow-up of an intervention involving the caregivers of elderly persons with mild dementia. Similar successes were reported in studies carried out in Thailand [28], Korea [29], and Japan [30].

According to the present study findings, the educational level of the elderly had a positive influence on the improvement of their MMSE score, whereas the female gender had a negative impact. The positive effect of education is in congruence with the findings of the study of Tse et al [31] where cognitive impairment showed a decrease with the number of years of education among the older adult community of Hong Kong. Concerning the gender effect, the better effect of the intervention among males might be due to that males have better short-term memory than females as mentioned by Conway et al [32]. However, on the contrary, Spector et al [33] showed that women had more improvements in memory, attention, and communication by using cognitive exercises compared to men. Meanwhile, Devraj [34] could not reveal any difference between males and females even after controlling for age and education. Hence, this issue of gender difference needs further study.

The present study demonstrated a gradual improvement in participants' MMSE scores throughout the follow-up period. This demonstrates the importance of continuity of the training on memory aids for longer time to booster the benefits. The finding is in congruence with Laffan et al [35] whose study in the United States demonstrated a significant decreasing trend in dementia severity, with better performance in memory, abstraction, and behavioral symptoms. Similar findings were shown in studies in Australia [36] and in Greece [37]. Lastly, the current study finding of better MMSE scores in the morning compared with the evening may be explained by the effect of the circadian rhythm as suggested by Valdez [38].

\section{Conclusion and Recommendations}

From the study findings, it can be concluded that training the caregivers of elderly people in mental stimulation and memory aids can improve the cognitive impairment among these persons. The findings corroborate the need for developing intervention programs that equip the caregivers of elderly people with non-pharmacological management strategies that enable them to improve their cognitive impairment. Periodic screening using simple testing is recommended for early detection of mental health problems in elderly people. Further research is needed to investigate the effectiveness of memory aids in improving the memory of elderly at different stages of dementia.

\section{References}

[1] Meijer W. A., van Boxtel M. P., Van Gerven P. W., van Hooren S. A., and Jolles J. (2009): Interaction effects of education and health status on cognitive change: a 6-year follow-up of the Maastricht Aging Study. Aging \& Mental Health; 13: 521529. 
[2] Coubard O.A., Ferrufino L., Boura M., Gripon A., Renaud M., and Bherer L. (2011): Atten-tional control in normal aging and Alzheimer's disease. Neuropsychology; 25:353-367.

[3] Fratiglioni L., and Qiu C. (2011): Prevention of cognitive decline in ageing: dementia asthe target, delayed onset as the goal. Lancet Neurology; 10: 778-779.

[4] McGuire L. C., Ford E. S., and Ajani U. A. (2006): Cognitive functioning as a predictor offunctional disability in later life. The American Journal of Geriatric Psychiatry; 14: 36-42.

[5] Plassman B. L., Langa K. M., Fisher G. G., Heeringa S. G., Weir, D. R., Ofstedal M. B., Burke J. R., Hurd M. D., Potter G. G., Rodgers W. L., Steffens D. C., McArdle J. J., Willis R. J., and Wallace R. B. (2008): Prevalence of cognitive impairment without dementia in the United States. Annals of Internal Medicine; 148: 427-W490.

[6] Petersen, R. C., Caracciolo, B., Brayne C., Gauthier S., Jelic $V$., and Fratiglioni L. (2014): Mild cognitive impairment: a concept in evolution. Journal of Internal Medicine; 275: 214228 .

[7] Petersen R. C., Roberts R. O., and Knopman D. S. (2009): Mild cognitive impairment: ten years later. Archives of Neurology; 66: 1447-1455.

[8] Langa K. M. and Levine D. A. (2014): The diagnosis and management of mild cognitive impairment: a clinical review. JAMA.; 312(23): 2551-61.

[9] Winblad B., Palmer K., Kivipelto M., Jelic V., Fratiglioni L., Wahlund L. O., Nordberg A., Bäckman L., Albert M., Almkvist O., Arai H., Basun H., Blennow K., De Leon M., Decarli C., Erkinjuntti T., Giacobini E., Graff C., Hardy J., Jack C., Jorm A., Ritchie K., Van Duijn C., Visser P., and Petersen R. C. (2004): Mild cognitive impairment - beyond controversies, towards a consensus: report of the International Working Group on Mild Cognitive Impairment. Journal of Internal Medicine; 256: 240-246.

[10] Coley N., Andrieu S., Gardette V., Gillette-Guyonnet S., Sanz C., Vellas B., and Grand A. (2008): Dementia prevention: methodological explantations for in consistent results. Epidemiologic Reviews; 30: 35-66.

[11] Mangialasche F., Kivipelto M., Solomon A., and Fratiglioni L. (2012): Dementia pre-vention: current epidemiological evidence and future perspective. Alzheimer's Research \& Therapy; 4: 6.

[12] Martin M., Clare L., Altgassen A. M., Cameron M. H., and Zehnder F. (2011): Cognition-based interventions for healthy older people and people with mild cognitiveimpairment. Cochrane Database Systematic Review; CD006220.

[13] Reijnders J., van Heugten C., van Boxtel M. (2012): Cognitive interventions in healthyolder adults and people with mild cognitive impairment: a systematic review. Ageing Research Reviews.

[14] Wilson R. S., Scherr P. A., Schneider J. A., Tang Y., and Bennett D. A. (2007): Relation of cognitive activity to risk of developing Alzheimer disease. Neurology; 69: 1911-1920.

[15] Folstein, M. F., Folstein, S. E. and McHugh, P. R. (1975): "Mini-Mental State": a practical method for grading the cognitive state of patients for the clinician Journal of Psychiatric Research, 189-198.
[16] World Health Organization [WHO], (2012): Alzheimer's disease international. Dementia: A public health priority.

[17] Delgado Derio C., Guerrero Bonnet S., Troncoso Ponce M., Araneda Yañez A., Slachevsky Chonchol A., and Behrens Pellegrino M. I. (2012): Memory, fluency, and orientation: a five-minute screening test for cognitive decline. Neurologia. 2013 Sep; 28(7): 400-7. doi: 10.1016/j.nrl.2012.10.001. Epub 2012 Dec 14.

[18] Hall L., Orrell M., Stott J., Spector A. Cognitive stimulation therapy [CST], (2013): Mar neuropsychological mechanisms of change. Int Psychogeriatr.; 25(3):479-89

[19] Lowenstein D. A., Acevedo A., Czaja S. J., and Duara R. (2004): Cognitive rehabilitation of mildly impaired Alzheimer disease patients on cholinesterase inhibitors. Am. J. Geriatr. Psychiatry; 395-402.

[20] Holthoff V. A., Marschner K., Scharf M., Steding J., Meyer S., Koch R., and Donix M. (2015): Effects of physical activity training in patients with Alzheimer's dementia: results of a pilot RCT study. PLoS One.; 10(4): e0121478. doi: 10.1371/journal.pone. 0121478. eCollection 2015.

[21] Alvaro L. (2012): Competency: general principles and applicability in dementia Neurologia; 27(5): 290-300. doi: 10.1016/j.nrl.2011.12.005. Epub 2012 Feb 17

[22] Tsantali E., Tsolaki M., and Economides D. (2009): The effects of a cognitive training program on trained and untrained cognitive functions of non demented elderly and Alzheimer's patients. International Journal of Psychosocial Rehabilitation; 77-98.

[23] Lacy M., Kaemmerer T., and Czipri S. (2015): Standardized mini-mental state examination scores and verbal memory performance at a memory center: implications for cognitive screening. Am J Alzheimers Dis Other Demen.; 30(2): 145-52.

[24] Haslam C., Moss Z., and Hodder K. (2010): Are two methods better than one? Evaluating the effectiveness of combining errorless learning with vanishing cues. J. Clin. Exp. Neuropsychol.; 32: 973-985.

[25] Belleville S., Clement F., Mellah S., Gilbert B., Fontaine F., and Gauthier S. (2011): Training-related brain Plasticity in subjects at risk of developing Alzheimer's disease. Brain; $1623-1634$

[26] Hampstead B. M., Stringer A. Y., Stilla R. F., Deshpande G., Hu X., Moore A. B., and Sathian K. (2011): Activation and effective connectivity changes following explicit-memory training for face- name pairs in patients with mild cognitive impairment: a pilot study. Neurorehabil Neural Repair; 210 222.

[27] Cruz T. J., Sá S. P., Lindolpho M. D., and Caldas C. P. (2015): Cognitive stimulation for older people with Alzheimer's disease performed by the caregiver. Rev Bras Enferm.; 68(3): 510-516.

[28] Lam L. C., Ong P. A., Dikot Y., Sofiatin Y., Wang H., Zhao M., Li W., Dominguez J., Natividad B., Yusoff S., Fu J. L., Senanarong V., Fung A. W., and Lai K. (2015): Intellectual and physical activities, but not social activities, are associated with better global cognition: a multi-site evaluation of the cognition and lifestyle activity study for seniors in Asia (CLASSA). Age Ageing.; 44(5): 835-40. 
[29] Cho M., Kim D., Chung J., Park J., You H., and Yang Y. (2015): Effects of a cognitive-enhancement group training program on daily living activities, cognition, and depression in the demented elderly. J Phys Ther Sci.; 27(3): 681-4.

[30] Nakatsuka M., Nakamura K., Hamanosono R., Takahashi Y., Kasai M., Sato Y., Suto T., Nagatomi R., and Meguro K. (2015): A Cluster Randomized Controlled Trial of Nonpharmacological Interventions for Old-Old Subjects with a Clinical Dementia Rating of 0.5: The Kurihara Project. Dement Geriatr Cogn Dis Extra.; 5(2): 221-32.

[31] Tse C. S., Chang J. F., Leung G. T., Fung A. W., Hau K. T., Chiu H. F., and Lam L. C. (2013): Effects of education on very mild dementia among Chinese people in Hong Kong: potential mediators in the Cantonese Mini-Mental State Examination tasks. Aging Ment Health.; 17(3): 310-8. doi: 10.1080/13607863.2012. 743962. Epub 2012 Nov 26.

[32] Conway, Andrew R. A.; Skitka, Linda J.; Hemmerich, Joshua A.; Kershaw, Trina C. (July 2009). "Flashbulb memory for 11 September 2001". Applied Cognitive Psychology 23(5): 605623. doi: 10.1002/acp.1497.

[33] Spector A., Aguirre E., Woods R. T., and Orrel M. (2013): Cognitive stimulation for dementia: a systematic review of the evidence of effectiveness from randomized controlled trials. Ageing Res Rev; 253-262.
[34] Devraj R., Singh VB, Ola V., Meena B. L., Tundwal V., and Singh K. (2014): Cognitive function in elderly population - An urban community based study in north-west Rajasthan. Journal, Indian Academy of Clinical Medicine; 15(2).

[35] Laffan A. J., Metzler-Baddeley C., Walker I., and Jones R. W. (2010): Making errorless learning more active: self-generation in an error free learning context is superior to standard errorless learning of face-name associations in people with Alzheimer's disease. Neuropsychol. Rehabil; 20: 197-211.

[36] Woods R. T., Whitaker R., Ballard C., Stafford J., Orrell M., Moniz-Cook E., Murray J., Knapp M., Carlton B. W., and Fossey J. (2013): Feasibility study of an optimized personcentered intervention to improve mental health and reduce antipsychotics amongst people with dementia in care homes: study protocol for a randomized Controlled trial; 13-14.

[37] Tsolaki M., Kounti F., Agogiatou C., Poptsi E., Bakoglidou E., and Zafeiropoulou M. (2011): Effectiveness of non pharmacological approaches in patients with mild cognitive impairment. Neurodegener Dis; 138-145.

[38] Valdez P., Ramírez C., and Garcia A. (2012): Circadian rhythms in cognitive performance: implications for neuropsychological assessment; 89. 